IdeAs

Idées d'Amériques

14 | 2019

Populismes dans les Amériques

\title{
Populismes dans les Amériques
}

Populism in the Americas

Populismos en las Américas

Luc Capdevila, François Vergniolle de Chantal et Jean-Christian Vinel

\section{OpenEdition}

\section{Journals}

Édition électronique

URL : http://journals.openedition.org/ideas/6816

DOI : $10.4000 /$ ideas.6816

ISSN : 1950-5701

Éditeur

Institut des Amériques

Référence électronique

Luc Capdevila, François Vergniolle de Chantal et Jean-Christian Vinel, « Populismes dans les

Amériques », IdeAs [En ligne], 14 | 2019, mis en ligne le 01 octobre 2019, consulté le 23 septembre

2020. URL : http://journals.openedition.org/ideas/6816 ; DOI : https://doi.org/10.4000/ideas.6816

Ce document a été généré automatiquement le 23 septembre 2020.

\section{cc) (†)}

IdeAs - Idées d'Amériques est mis à disposition selon les termes de la licence Creative Commons

Attribution - Pas d'Utilisation Commerciale - Pas de Modification 4.0 International. 


\title{
Populismes dans les Amériques
}

\author{
Populism in the Americas \\ Populismos en las Américas
}

Luc Capdevila, François Vergniolle de Chantal et Jean-Christian Vinel

$1 \mathrm{Au}$ cours des dix dernières années, le populisme s'est affirmé comme une notion souvent clef dans l'analyse de l'évolution politique et sociale d'un grand nombre de pays européens ou américains. Son usage reste cependant très largement négatif car il désigne le plus souvent un appel direct au peuple basé sur le ressentiment contre les élites et/ou certains groupes sociaux, tout en prônant un arsenal de mesures «simples » et démagogiques. Il est d'ailleurs principalement utilisé pour dénoncer ses ennemis et il y a fort peu de responsables politiques - à l'exception par exemple de Jean-Luc Mélenchon de la France Insoumise, d'Hugo Chavez, ou encore des leaders de Podemos en Espagne (Chazel L., 2019) - qui revendiquent ce label avec fierté1. En d'autres termes, le populisme est perçu d'abord et avant tout comme une forme dégradée de la vie démocratique. C'est dans ce sens que l'analyse Pierre Rosanvallon (2011) en y voyant une triple simplification: d'abord une simplification politique en opposant un peuple fantasmé, homogène, à des élites corrompues; ensuite une simplification institutionnelle en rejetant tout contrepoids face à la volonté populaire ; et enfin une simplification sociale, qui attribue au peuple une identité claire et définie ${ }^{2}$.

2 Mais le populisme est aussi une notion dont les pratiques et les styles sont très différents. Dans les Amériques, le populisme a ainsi été invoqué ces derniers temps pour désigner des courants et des sensibilités politiques très variés, en référence à des mouvements, des acteurs, voire des gouvernements tels que Occupy Wall Street, le Tea Party, Bernie Sanders, ou encore Donald Trump. Au Sud, c'est notamment le cas des régimes que l'on qualifie aussi de national-populaires en Amérique latine : le Venezuela chaviste, la Bolivie dirigée par Evo Morales, l'Argentine sous les gouvernements Kirchner...; tandis que le terme de populisme est également utilisé pour désigner des courants ultra-conservateurs, tel celui incarné par Jair Bolsonaro, l'actuel président du Brésil, ou les gouvernements néo-libéraux des années 1990 (Fujimori au Pérou, Collor de Mello au Brésil, Bucaram en Équateur...). Ces derniers, tout en étant dirigés par des «hommes forts » élus dans des contextes de crise politique, avaient renoué avec des 
pratiques d'appel au peuple, d'immédiation avec la société selon de nouvelles procédures (cathodiques, par exemple dans les années 1990, numériques aujourd'hui), de pouvoir fortement incarné, en étant parfois directement issu des anciennes organisations populistes (Menem en Argentine). Mais en abandonnant les politiques redistributives et d'intervention économique caractéristiques des «vieux populismes en Amérique latine", ils participeraient des "néopulismes" définis à partir de "la fusion entre populisme et néolibéralisme » qu'ils négocient (Freindenberg F., 2007).

Dans ces conditions, nombreux sont ceux qui dénient à la notion de populisme tout contenu: si le populisme n'est pas tant une idéologie, il se réduit dès lors à ses multiples usages. L'ubiquité masque en effet une certaine confusion conceptuelle (Mudde C., 2007, Hermet G., 2001, Taguieff P.-A., 1997, Laclau E., 2008), d'autant que le populisme semble être l'ombre de la démocratie (Arditi B., 2004, Canovan M., 1999). Si l'actualité du populisme témoigne du sentiment que les démocraties vacillent sous le poids de demandes économiques, sociales, identitaires et sécuritaires qui, souvent, prennent la forme d'une dénonciation des élites et d'une défense d'un peuple vertueux, sa substance reste floue. D'une part, parce que deux niveaux de discours - celui des acteurs et celui des analystes, qu'ils viennent des sciences sociales ou politiques - se mélangent dans les débats. D'autre part, parce que le caractère transnational et la diversité du phénomène tendent à masquer les différences séparant les cas nationaux. L'histoire du populisme en Europe est notamment fort différente de celles des ÉtatsUnis, du Canada et de l'Amérique latine. Sur le Vieux continent, le populisme renvoie en règle générale à des mouvements d'extrême-droite (Mudde C., 2007), ou, comme le disait George Lavau (1970), à une «fonction tribunitienne » des partis de gauche. En revanche, aux États-Unis, berceau du Parti éponyme, il constitue un langage politique traduisant une passion égalitaire issue de la révolution américaine (Kazin M., 1995). Quant à l'Amérique latine, souvent considérée comme sa «terre d'élection " (Hermet G., 2001), qui en ferait le "paradigme du populisme moderne » (Dorna A., 1999), il désigne d'abord un "populisme de gouvernement» plus que de "dénonciation » ou même «d'opposition" (Rosanvallon P., 2011), dont la diversité des expériences a profondément marqué le xxe siècle du sous-continent (Marques Pereira B., Garibay D., 2011), et dont les héritages restent forts dans les premières décennies du iiie millénaire. Mais la «fréquence » et "l'imprécision » du concept nécessitent d'affiner le contexte de son utilisation conduisant à qualifier une pluralité de mouvements et de régimes historiquement situés, et ce d'autant plus que, à quelques exceptions près, "personne ne se dit ou ne se prétend populiste » (Rouquié A., [1987] 1998).

Aux États-Unis, le populisme a de profondes racines qui remontent à la fondation du pays. D'un point de vue théorique, les affinités entre le premier pays dont la constitution de 1787 stipule «We The People...Do » et le populisme, qui revendique les principes de base de la démocratie (règle majoritaire et souveraineté populaire), confirment à quel point le populisme suit en effet la démocratie comme son ombre (Arditi B., 2004). Mais la relation entre les deux est néanmoins pleine de tensions, ce dont témoigne abondamment l'histoire des Etats-Unis. Dès les années 1780 , les Pères fondateurs fédéralistes dénonçaient la "tyrannie de la majorité ", une expression qui recoupe largement l'emploi contemporain de «populisme». C'est pourquoi la constitution pensée par James Madison et les autres délégués de Philadelphie multipliait les "checks and balances" afin de contrer cette tyrannie tant redoutée. Depuis lors, quantité de mouvements populistes - de droite comme de gauche - ont critiqué ces «contrepoids» institutionnels comme autant de moyens de museler la 
parole du peuple. Comme l'écrivent Yves Mény et Yves Surel (2002), les mouvements populistes partagent l'idée que la démocratie ne signifie que le pouvoir du peuple. Ce faisant, ils exploitent volontiers le fossé entre les idéaux démocratiques - que l'on songe par exemple à la fameuse citation de Lincoln en 1863 louant le " government of, by, for the people » - et le fonctionnement élitiste de la démocratie représentative, telle qu'analysée par une série de politistes et de théoriciens "réalistes ", allant de Joseph Schumpeter, à Robert Dahl, Giovanni Sartori, sans oublier Bernard Manin. Cette critique est lancinante dans la vie politique américaine. Elle revient à intervalles réguliers avec néanmoins comme caractéristique singulière que ces mouvements populistes ne critiquent jamais la constitution. Ils peuvent appeler à sa réforme (c'est le cas à gauche), à son respect (c'est le cas à droite), mais jamais à son abolition.

5 La particularité états-unienne réside aussi dans le fait que le populisme y est historiquement associé à des mouvements progressistes, à l'inverse des démocraties européennes où la très grande majorité des populismes sont de droite. De fait, c'est un journaliste du Kansas qui, en 1890, utilise pour la première fois le terme "populisme " en référence au People's Party, un tiers parti, qui se transforme en force politique majeure avec plus de $8 \%$ des suffrages à l'élection présidentielle de 1892. L'émergence de ce mouvement de protestation au sein de l'Amérique rurale, à un moment de transition entre un capitalisme de propriétaires individuels et un capitalisme dominé par les grandes entreprises, a nourri un large débat historiographique. En s'appuyant sur l'Ecole de Francfort, Richard Hofstadter présentait le populisme comme une réaction irrationnelle et pathologique contre la modernisation économique ; il illustrait à ses yeux le "style paranoïaque" de la vie politique américaine dont la droite libertarienne, incarnée par Goldwater, illustrait le potentiel (Hofstadter R., 1955, 1964). Cette thèse, néanmoins, fut mise à mal par des historiens qui, dans le sillage de Lawrence Goodwyn, soulignaient l'imprégnation démocratique d'un mouvement disposant d'une forte dimension associative et dont l'influence législative fut également visible sur les premières lois encadrant le travail à l'usine, mais aussi sur certaines lois du New Deal (Goodwyn L., 1978, Postels C., 2007, Sanders E., 1999). Certes, dans les années 1930, le populisme prit des accents menaçants, voire fascistes, avec Huey Long et Father Coughlin, mais comme le montre Alan Brinkley, il conservait une dimension démocratique et poussa Roosevelt et les New Dealers à adopter des réformes sociales importantes (Brinkley A., 1982).

De fait, toute la difficulté de l'analyse du populisme aux Etats-Unis tient à sa grande complexité au cours du xxe siècle. Pour Michael Kazin, il faut en fait opérer un distinguo entre le People's Party et le langage populiste (au sens que l'historien Gareth Stedman Jones donne à ce terme) qu'il légua à la vie politique américaine. Ce langage, qui oppose un peuple vertueux et travailleur représentant l'essence de l'Amérique à une élite de parasites la menaçant, opère, selon Kazin, comme un substitut à une rhétorique de classe assumée, mais les démocrates et la gauche américaine n'en ont guère l'apanage : le populisme devient, à partir des années 1950, un rouage essentiel à la reconfiguration de la droite américaine, qui le met à profit pour critiquer les élites intellectuelles progressistes et leurs projets d'ingénierie sociale, attirant ainsi une partie de l'électorat ouvrier. Dans un contexte de tensions croissantes liées aux combats autour de la discrimination raciale et sexuelle dans l'emploi, aux guerres culturelles, et à la crise économique dans les années 1970, le populisme sert la défense d'une Amérique blanche et conservatrice. De George Wallace à Ronald Reagan et Pat Buchanan, ce populisme de droite fait l'éloge de l'Amérique au travail et critique 
parfois les lobbys de K Street, mais son contenu idéologique et son rapport aux classes sociales reste ambigu, car il débouche sur des réformes qui reflètent les intérêts des élites économiques et participe ainsi à l'essor du néolibéralisme (Kazin M., 1995, 2017, Frank T., 2004).

7 Au total, la prégnance du populisme aux Etats-Unis illustre ainsi l'absence de toute tradition marxiste d'ampleur - rendue encore plus marginale après la révolution bolchévique, puis la Guerre froide - et le discrédit frappant les arguments liés à la classe qui en a été la conséquence. Cependant, sa malléabilité et son ancrage à droite depuis près de soixante ans témoignent sans doute également de la nécessité de cerner ses liens avec l'histoire sociale et politique de l'Amérique péri-urbaine et l'anti-étatisme des classes moyennes qui y forment l'assise électorale du Parti républicain (McGirr L., 2001).

8 Pour ces raisons, insérer Donald Trump dans la trame du populisme américain n'est guère aisé, ce qui explique sans doute que bien des observateurs préfèrent revenir à la thèse de Hofstadter, qui a retrouvé une certaine actualité. Nombreux furent ceux à décrier Trump comme une anomalie de la vie politique américaine, qui n'avait réussi à conquérir le Parti républicain, puis la présidence, qu'à la suite d'un concours de circonstances. Le phénomène Trump est néanmoins bien plus complexe que ses discours ne le laissent penser, notamment parce que son électorat, en dépit de basculements au niveau local dans la rust belt, recoupe l'électorat traditionnel du Parti républicain, mais aussi parce que ses positions contre l'immigration et en faveur du protectionnisme font écho à la politique menée par le Parti républicain à la fin du XIXe siècle. Après des années de mobilisation conservatrice volontiers populiste, Trump peut certes représenter une forme d'épuisement de cette "révolution conservatrice " des dernières décennies. Mais il constitue surtout un parfait exemple de ce que P-A Taguieff définit comme un " populisme-attitude » (1997, p. 15), indépendant des visions ou des traditions plus ou moins structurées, politiquement indéterminé, mais idéalisant le " populaire ». Qui plus est, l'administration Trump constitue une étape cruciale dans l'histoire américaine du populisme en ceci que ce dernier n'est plus seulement un mouvement extra-institutionnel, mais, bien au contraire, est installé au cœur du pouvoir.

9 En Amérique latine, en revanche, l'expérience gouvernementale du populisme est bien plus importante. Le contexte d'émergence du terme de populisme en Amérique latine est lié au développement des sciences sociales dans le sous-continent, à la fin des années 1950/début des années 1960. Sociologues et politistes se sont alors appropriés le concept pour analyser les régimes autoritaires et nationalistes à l'origine des politiques de régulation et de redistribution, caractéristiques de la séquence qui venait de s'écouler (Quattrocchi-Woisson D., 1997). D'abord appliqué au péronisme en Argentine (Germani G., 1962 ; di Tella T., 1964) et au gétulisme au Brésil (Cardoso F., Weffort F. (eds.), 1970), le concept a été rapidement étendu à l'échelle du sous-continent pour appréhender la conjoncture des années 1920/1960, au cours de laquelle des partis (tels l'APRA au Pérou, le parti fébrériste au Paraguay, le MNR en Bolivie), des leaders n'ayant pas accédé aux affaires (Cesar Sandino au Nicaragua, Jorge Eliécer Gaitán en Colombie), des gouvernements (le Mexique post-révolutionnaire, Gualberto Villaroel en Bolivie, Velasco Ibarra en Equateur), ont incarné les réponses à la crise institutionnelle (fiction démocratique) et économique (modèle agro-exportateur) des républiques oligarchiques. Le dénominateur commun fut d'inscrire le «peuple» au cœur de leurs 
discours de mobilisation, qu'il ait été celui des «sans chemise» péronistes en Argentine, des « oubliés » en Équateur sous Velasco Ibarra, des " pauvres » au Brésil dirigé par Vargas, des « pieds nus » (pynandí) colorados au Paraguay.

10 Au-delà de la diversité des expériences nationales vécues, plusieurs éléments permettent de caractériser les singularités et l'historicité de ces populismes latinoaméricains. Généralement, les dirigeants ont été portés par une dynamique démocratique associée à l'extension du suffrage. Ils impulsèrent des politiques développementistes fondées sur la régulation étatique et la redistribution sociale. Les discours de mobilisation ont reposé sur une double polarisation: le peuple contre les élites, la nation contre les impérialismes. Ce sont en effet des régimes de mobilisation en phase avec les mouvements sociaux, fondés sur un fort encadrement syndical et/ou partisan. Enfin, la plupart de ces régimes se sont engagés dans une transformation limitée des structures socio-économiques, la nationalisation des secteurs stratégiques n'ayant que très rarement la réforme agraire pour corollaire. De sorte qu'ils ont abouti à un équilibre fragile en organisant l'«État de compromis", censé préserver la propriété des élites tout en répondant en partie aux attentes sociales des secteurs populaires et des classes moyennes.

11 Régimes de "transition " qui s'efforcèrent "d'intégrer les secteurs populaires dans l'ordre politique» (Rouquié A., 1998), ils ont marqué une étape essentielle dans la construction du processus démocratique en Amérique latine. Les convocations électorales et l'extension du suffrage ont accompagné l'accession au pouvoir de Perón en Argentine en 1946 et 1952, d'Estenssoro en Bolivie en 1952, le retour de Vargas au Brésil en 1951. En ce sens, ces gouvernements "populistes" latino-américains ont généralement engagé et porté les politiques indigénistes du premier vingtième siècle, dans la perspective de tisser la nation, de cristalliser une identité nationale enracinée dans le territoire et fondée sur l'autochtonie, mais également d'intégrer l'ensemble des secteurs populaires, qu'ils fussent indiens, afro-latino-américains (Andrews G. R., 2007), voire issus de l'immigration. Les conflictualités des années 1960 mettent en évidence les limites idéologiques des populismes historiques latino-américains. La voie révolutionnaire cubaine, la volonté de changement social dont la réforme agraire fut un des maitres mots, ont rompu l'équilibre obtenu avec l'État de compromis et fracturé les mouvements, dont l'unité reposait parfois sur la seule expression de la fidélité à l'égard du leader; tandis que les dictatures de guerre froide des années 1970 ont affirmé vouloir en finir avec l'« aberration populiste » (Quattrocchi Woisson D., 1997).

Quant aux gouvernements réformistes des années 2000/2010, qualifiés à nouveau de "populistes" par leurs détracteurs (Panizza F., 2008), ils ne peuvent être considérés que dans une mesure toute relative comme étant les héritiers des régimes du siècle passé ; la filiation avec les «vieux populismes» étant plus encore problématique lorsqu'il s'agit des «néopulismes » contemporains articulant appel au peuple et mise en œuvre des politiques néolibérales et de soutien aux grandes firmes. Néanmoins, les premiers furent d'abord le fruit de la consolidation démocratique, qui mit fin aux dictatures de guerre froide, et de l'alternance qui s'imposa à la suite de la crise sociale provoquée par deux décennies de politiques d'ajustement structurel et de dérégulation néolibérale. Au-delà des politiques redistributives et d'une action réelle contre la pauvreté engagée dans les années 2000 par les gouvernements de gauche, certains dirigeants ont certes renoué avec un pouvoir personnaliste, clivant, et des pratiques d'immédiation avec «le peuple", mais tout en articulant l'action gouvernementale 
avec des organisations de base, qu'elles soient associatives, communautaires, syndicales ou communales. Ces derniers éléments peuvent caractériser ces nouveaux "populismes" latino-américains. Mais à la différence de leurs prédécesseurs polyclassistes et "national-populaires", ils ont fonctionné dans un contexte démocratique consolidé et se sont positionnés nettement à gauche sur l'échiquier politique national et international.

Dès lors, existe-t-il un modèle populiste? américain? Ou pourrait-on davantage convoquer un laboratoire américain, avec des expériences populistes diverses et de nombreuses singularités nationales, qui interrogent en particulier: les liens entre populisme et construction démocratique; entre populisme et électoralisme; entre populisme et participation?

14 Ce numéro d'IdeAs propose un éclairage décalé sur la question en montrant, à partir d'études localement situées, comment les historiens, les sociologues et les politistes travaillant sur les Amériques du Sud et du Nord mobilisent la notion de populisme en regard de phénomènes sociaux, politiques et économiques dont elle permet de rendre raison. Qu'est-ce qui, dans une démocratie, différencie le populisme de l'appel aux suffrages des électeurs, et notamment celui des classes populaires ? Comme l'écrivaient Yves Mény et Yves Surel (2002), les mouvements populistes s'expriment et se comportent en effet comme si la démocratie se réduisait au pouvoir du peuple. Mais ce diagnostic général demande à être affiné, car les populismes, bien loin d'être en opposition systématique avec les sophistications de la démocratie libérale et représentative (Manin, B., 2005), se déclinent tout au long d'un continuum. Le populisme peut parfois prendre des formes qui sont toxiques pour la démocratie, mais il peut aussi, sous d'autres formes, réaliser ce que James Morone (1990) appelle le « vœu démocratique" (Democratic Wish). C'est pourquoi, et comme l'explique Joseph Lowndes dans son chapitre du Oxford Handbook of Populism (2017), « il est sans doute préférable d'analyser le populisme par ce qu'il fait plutôt que par ce qu'il est » ([it is] perhaps better then to analyze not what populism was but what populism did). Dans cette perspective, l'objectif de ce dossier est de contribuer à identifier des critères populistes à partir d'expériences et d'analyses tirées du continent américain, du Canada au Cône Sud.

\section{BIBLIOGRAPHIE}

Andrews, George Reid, Afro-Latinoamérica, 1800-2000, Madrid/Frankfurt am Main, Iberoamericana/Vervuert, 2007.

Arditi, Benjamin, «Populism as a Spectre of democracy », Political Studies, vol. 52, n 1, mars 2004, p. 135-143.

Brinkley, Alan, Voices of Protest : Huey Long, Father Coughlin and the Great Depresssion, New York : Knopf, 1982. 
Canovan, Margaret, « Two Strategies for the Study of Populism », Political Studies, vol. $30, \mathrm{n}^{\circ} 4$, 1982.

Cardoso, Fernando Enrique y Weffort, Francisco, (Eds.), América latina. Ensayos de interpretación sociológico-política, Santiago de Chile, Editorial Universitaria, 1970.

Chazel, Laura, « De l'Amérique latine à Madrid : Podemos et la construction d'un « populisme de gauche », Revue Pôle Sud, n50, 2019, p. 121-138.

Di Tella, Torcuato, El sistema político argentino y la clase obrera, Buenos Aires, Eudeba, 1964.

Dorna, Alexandre, Le populisme, Paris PUF, 1999.

Frank, Thomas, What's the Matter With Kansas? New York, Holt, 2004.

Freidenberg, Flavia, La tentación populista. Una vía al poder en América latina, Madrid, Editorial Sintésis, 2007.

Germani, Gino, Política y sociedad en una época de transición. De la sociedad tradicional a la sociedad de masas, Buenos Aires, Paidós, 1962.

Goodwyn, Lawrence, Democratic Promise : The Populist Moment in America, New York, Oxford UP, 1976.

Hermet, Guy, Les populismes dans le monde, Paris, Fayard, 2001.

Hofstadter, Richard, The Age of Reform : From Bryan to FDR, New York, Knopf, 1955.

Hofstadter Richard, The Paranoid Style in American Politics, New York, Vintage, 1964.

Kaltwasser, Cristóbal Rovira \& al. (eds.). The Oxford Handbook of Populism, Oxford : Oxford University Press, 2017

Kazin, Michael, « The Populist Persuasion », Ithaca, Cornell UP, 1995. Nouvelle édition en 2017.

Laclau, Ernesto, La raison populiste, Paris, Seuil, 2008.

Lowndes, Joseph, Populism in the United States", p. 232-246, in Cristobal R. Kaltwasser, et al., The Oxford Handbook of Populism, Oxford, Oxford University Press, 2017.

McGirr, Lisa, Suburban Warriors, The Origins of the New American Right, Princeton, Princeton UP, 2001.

Marques-Pereira, Bérengère, Garibay, David, La politique en Amérique latine. Histoires, institutions et citoyennetés, Paris, Armand Colin, 2011.

Manin, Bernard, The Principles of Representative Government, Cambridge, Mass., Cambridge University Press, 2005.

Morone, James, The Democratic Wish. Popular Participation and the Limits of American Government, New York, Basic Books, 1990.

Mudde, Cas, Populist Radical Right Parties in Europe, Cambridge,UK, Cambridge UP, 2007.

Panizza, Francisco, « Fisuras entre Populismo y Democracia en América Latina », Stockholm Review of Latin American Studies, $n^{\circ}$ 3, 2008, p. 81-93.

Postels, Charles, The Populist Vision, New York, Oxford, 2007.

Quattrocchi-Woisson, Diana, « Les populismes latino-américains à l'épreuve des modèles d'interprétation européens ", Vingtième siècle, revue d'histoire, n 56, 1997, p. 161-183.

Rosanvallon, Pierre, « Penser le populisme », laviedesidees.fr, 2011. 
Rouquié, Alain, Amérique latine. Introduction à l'extrême-Occident, Paris, Seuil, 1998 [1987 1ère édition]

Sanders, Eilzabeth, Roots of Reform, Farmers, Workers, and theAmerican State, 1877-1917, Chicago, The University of Chicago Press, 1999.

Surel,Yves, Mény, Yves, « Populism : The Pathology of Democracy? », in Democracies and the Populist Challenge, London : Palgrave, 2002, 3-6.

Taguieff, Pierre-André, « Le populisme et la science politique : du mirage conceptuel aux vrais problèmes ", Vingtième Siècle, n 56, octobre-décembre 1997, p. 4-33.

Wiles, Peter, « A Syndrome, Not a Doctrine: Some Elementary Theses on Populism », in Ghita Ionescu, Ernest Gellner, ed., Populism. Its Meaning and National Characteristics, London, Weidenfeld and Nicolson, 196

\section{NOTES}

1. «Mélenchon, le populisme assumé », France Inter, L'édito politique, 29 août 2017 : https:// www.franceinter.fr/emissions/l-edito-politique/l-edito-politique-29-aout-2017.

2. Pierre Rosanvallon, « Penser le populisme », 27 septembre 2011 : http://www.laviedesidees.fr/ Penser-le-populisme.html.

\section{AUTEURS}

\section{LUC CAPDEVILA}

Luc Capdevila (UMR CNRS 6051 ARENES) est professeur d'histoire contemporaine à l'université Rennes 2. Spécialiste d'histoire sociale et des représentations, ses recherches portent plus particulièrement sur les dynamiques sociales et culturelles des sociétés en guerre en Amérique latine. Auteur de plusieurs ouvrages il a publié notamment : Una guerra total: Paraguay, 1864-1870. Ensayo de historia del tiempo presente, Asunción/Buenos Aires, Editorial Sb/Centro de Estudios Antropológicos de la Universidad Católica Nuestra Señora de la Asunción, 2010, 544 pages ; Los hombres transparentes. Indígenas y militares en la guerra del Chaco, 1932-1935, en colaboración con Isabel Combes, Nicolás Richard y Pablo Barbosa, Cochabamba, Instituto de misionología (UCB), col. Scripta Autochtona/Historia Indígena de las Tierras Bajas, 2010, 230 pages ; Le passé des émotions. D'une histoire à vif, Amérique latine et Espagne, Rennes, PUR, 2014, 204 pages, coordonné avec Frédérique Langue.

\section{FRANÇOIS VERGNIOLLE DE CHANTAL}

François Vergniolle de Chantal est politiste et professeur de civilisation américaine à l'Université de Paris (LARCA - UMR 8225). Il travaille sur les institutions politiques américaines, notamment le Congrès, les partis politiques et leur évolution, ainsi que les traditions politiques. Ses ouvrages les plus récents sont L'impossible présidence impériale (Editions du CNRS, 2016) et, en co-direction avec Agnès Alexandre-Collier, Leadership and Uncertainty Management in Politics: Leaders, Followers 
and Constraints in Western Democracies (Palgrave, 2015). Il publie en 2020 un ouvrage collectif intitulé Obama's Fractured Legacy: The Politics and Policies of an Embattled Presidency chez Edinburgh University Press. Il a co-dirigé (avec Alexandra de Hoop Scheffer) la revue Politique Américaine de 2012 à 2019.

http://www.larca.univ-paris-diderot.fr/membre/vergniolle-de-chantal-francois/

\section{JEAN-CHRISTIAN VINEL}

Jean-Christian Vinel (LARCA-CNRS) est Maître de conférences en histoire et civilisation des EtatsUnis à l'Université de Paris. Spécialiste d'histoire sociale et politique, il est l'auteur de plusieurs ouvrages, notamment The Employee: A Political History, Philadelphie, The University of Pennsylvania Press, 2013 ; Conservatisme en mouvement. Une approche transnationale. Paris, Presses de l'EHESS, 2016 (co-dirigé avec Clarisse Berthezène) et Liberal Orders: The Political Economy of the New Deal and Its Discontents, Philadelphie, University of Pennsylvania Press, 2020 (co-dirigé avec Nelson Lichtenstein et Romain Huret). Il rédige actuellement un ouvrage intitulé Reaction At Work: The Right, Labor and the Making of the New Gilded age, sous contrat avec Oxford University Press. 